\title{
Aldosterone Inhibits In Vitro Myogenesis by Increasing Intracellular Oxidative Stress via Mineralocorticoid Receptor
}

\author{
Jin Young Lee ${ }^{1}$, Da Ae Kim ${ }^{1}$, Eunah Choi ${ }^{1}$, Yun Sun Lee ${ }^{1}$, So Jeong Park ${ }^{1}$, Beom-Jun Kim² \\ ${ }^{1}$ Asan Institute for Life Sciences, ${ }^{2}$ Division of Endocrinology and Metabolism, Asan Medical Center, University of Ulsan \\ College of Medicine, Seoul, Korea
}

Background: Despite clinical evidence indicating poor muscle health in subjects with primary aldosteronism (PA), it is still unclear whether the role of aldosterone in muscle metabolism is direct or mediated indirectly via factors, such as electrolyte imbalance or impaired glucose uptake. As one approach to clarify this issue, we investigated the effect of aldosterone on in vitro myogenesis and the potential mechanism explaining it.

Methods: Myogenesis was induced in mouse $\mathrm{C} 2 \mathrm{C} 12$ myoblasts with $2 \%$ horse serum. Immunofluorescence, quantitative reversetranscription polymerase chain reaction, Western blot, viability, and migration analyses were performed for experimental research.

Results: Recombinant aldosterone treatment suppressed muscle differentiation from mouse $\mathrm{C} 2 \mathrm{C} 12$ myoblasts in a dose-dependent manner, and consistently reduced the expression of myogenic differentiation markers. Furthermore, aldosterone significantly increased intracellular reactive oxygen species (ROS) levels in myotubes, and treatment with N-acetyl cysteine, a potent biological thiol antioxidant, reversed the decrease of myotube area, myotube area per myotube, nucleus number per myotube, and fusion index due to aldosterone through decreasing oxidative stress. A binding enzyme-linked immunosorbent assay confirmed that mineralocorticoid receptor (MR) interacted with aldosterone in $\mathrm{C} 2 \mathrm{C} 12$ myoblasts, while eplerenone, an MR inhibitor, blocked aldosterone-stimulated intracellular ROS generation during myogenesis and markedly attenuated the suppression of in vitro myogenesis by aldosterone.

Conclusion: These findings support the hypothesis that hypersecretion of aldosterone, like PA, directly contributes to muscular deterioration and suggest that antioxidants and/or MR antagonists could be effective therapeutic options to reduce the risk of sarcopenia in these patients.

Keywords: Aldosterone; Sarcopenia; Muscle development; Receptors, mineralocorticoid; Oxidative stress

\section{INTRODUCTION}

Sarcopenia is a progressive skeletal muscle disorder characterized by the accelerated loss of muscle mass and function [1,2].

Received: 16 May 2021, Revised: 12 June 2021, Accepted: 15 June 2021

Corresponding authors: So Jeong Park

Asan Institute for Life Sciences, Asan Medical Center, University of Ulsan College of Medicine, 88 Olympic-ro 43-gil, Songpa-gu, Seoul 05505, Korea Tel: +82-2-3010-8718, Fax: +82-2-3010-6962, E-mail: sjpark99@hanmail.net

Beom-Jun Kim

Division of Endocrinology and Metabolism, Asan Medical Center, University of Ulsan College of Medicine, 88 Olympic-ro 43-gil, Songpa-gu, Seoul 05505, Korea Tel: +82-2-3010-5876, Fax: +82-2-3010-6962, E-mail: umkbj0825@amc.seoul.kr
This condition has recently drawn particular attention due to the increased likelihood of negative health outcomes, such as frailty, falls, physical weakness, poor quality of life, and mortality $[3,4]$, and has been officially classified as a disease, reflecting

Copyright $\odot 2021$ Korean Endocrine Society

This is an Open Access article distributed under the terms of the Creative Commons Attribution Non-Commercial License (https://creativecommons.org/ licenses/by-nc/4.0/) which permits unrestricted non-commercial use, distribution, and reproduction in any medium, provided the original work is properly cited. 
its clinical significance [5]. Although the term "sarcopenia" was first used to describe age-related muscular decline [6], increasing knowledge about muscle metabolism has revealed that sarcopenia could be attributable to heterogeneous causes, including systemic inflammatory diseases, malignancy, organ failure, or medications $[2,3,7]$. This secondary sarcopenia involving specific causes other than aging is particularly important from a clinical perspective because of its potential reversibility. Therefore, it is critically important to continuously pursue efforts to identify the modifiable causes of sarcopenia in order to extend the healthy lifespan in old age and to reduce the associated unnecessary economic burden on national healthcare systems.

Primary aldosteronism (PA) refers to inappropriate and autonomous hypersecretion of aldosterone from the adrenal glands $[8,9]$. It is regarded as a good human model to elucidate the role of excess aldosterone in the pathogenesis of diverse diseases. PA accounts for approximately $10 \%$ of hypertensive patients and is known to contribute to end-organ damage, especially in kidney, heart, and carotid artery [10,11]. Accumulating evidence now indicates that aldosterone can deleteriously affect muscle phenotypes as well. An animal study showed that the infusion of aldosterone-induced skeletal muscle apoptosis in rats [12]. Furthermore, compared with subjects with nonfunctional adrenal incidentaloma, those with PA had low skeletal muscle mass, leading to an increased risk of sarcopenia [13]. However, it is still unclear whether the role of aldosterone in muscle metabolism is direct or mediated indirectly by factors, such as electrolyte imbalance or impaired glucose uptake [9,14-16]. As one approach to clarify this issue, we investigated the direct effect of aldosterone on in vitro myogenesis and the potential mechanism explaining it.

\section{METHODS}

\section{Cell culture and reagents}

Mouse $\mathrm{C} 2 \mathrm{C} 12$ myoblasts were purchased from the American Type Culture Collection (ATCC, Manassas, VA, USA) and maintained in Dulbecco's Modified Eagle's Medium (DMEM) supplemented with $15 \%$ fetal bovine serum (FBS), $20 \mathrm{mM}$ hydroxyethyl piperazine ethane sulfonic acid (HEPES), $2 \mathrm{mM} \mathrm{L}$ glutamine, $100 \mathrm{U} / \mathrm{mL}$ penicillin, and $0.1 \mathrm{mg} / \mathrm{mL}$ streptomycin (Life Technologies Corp., Carlsbad, CA, USA) at $37^{\circ} \mathrm{C}$ in a humidified atmosphere containing $5 \% \mathrm{CO}_{2}$. To induce myogenesis, cells were grown to $90 \%$ confluency in maintenance media and switched to differentiation media (DMEM with $2 \%$ horse serum) for 3 or 4 days of culture. Recombinant aldosterone
(A9477), eplerenone (E6657), and $N$-acetyl-L-cysteine (NAC, A9165) were purchased from Sigma-Aldrich (St. Louis, MO, USA).

\section{Immunofluorescence}

Differentiated $\mathrm{C} 2 \mathrm{C} 12$ cells were fixed in $4 \%$ paraformaldehyde (PFA) for 15 minutes, washed twice with phosphate-buffered saline (PBS), permeabilized in $0.01 \mathrm{M}$ sodium citrate buffer containing $0.1 \%$ Triton X-100 for 10 minutes, and washed twice with PBS. The cells were blocked with $2 \%$ bovine serum albu$\min$ (BSA) in PBS for 1 hour and subsequently incubated with anti-myosin heavy chain (MyHC) antibody (MF20, Developmental Studies Hybridoma Bank, Iowa City, IA, USA) at $4^{\circ} \mathrm{C}$ overnight. The cells were incubated with Alexa Fluor 555-conjugated secondary antibodies (1:1,000 dilution, Cell Signaling, Danvers, MA, USA) for 1 hour and washed with $0.2 \%$ Tween20 in PBS. Thereafter, they were incubated with 4,6-diamidino2-phenyindole (DAPI, 1:10,000 dilution, Sigma-Aldrich) for 2 minutes and washed with PBS. The samples were mounted using Fluoromount G (Southern Biotech, Birmingham, AL, USA) and images were captured using a fluorescence microscope (Carl Zeiss, Jena, Germany). The area of the MyHC-stained myotubes was calculated using the ZEN 2 (blue edition) software (Carl Zeiss). Fusion index (\%) was calculated using the following equation: $100 \times$ number of nuclei in $\mathrm{MyHC}^{+}$myotubes per total number of nuclei in $\mathrm{MyHC}^{+}$myocytes and myotubes [17].

\section{Quantitative reverse-transcription polymerase chain reaction}

Total RNA was extracted using TRIzol reagent (Invitrogen, Carlsbad, CA, USA), in accordance with the manufacturer's protocol. After first-strand cDNA synthesis with the Superscript III First-Strand Synthesis System (Invitrogen) using oligo dT primers, quantitative reverse-transcription polymerase chain reaction was performed in triplicate on a Light Cycler 480 SYBR Green I Master (Roche, Mannheim, Germany) [18]. The primers for myogenin (NM_031189.2), myocyte enhancer factor 2C (Mef2C; NM_001170537.1), and MyHC (NM_001013397.2) were obtained from Applied Biosystems (Foster City, CA, USA). The threshold cycle $(\mathrm{Ct})$ value for each gene was normalized to the Ct value of 18S rRNA (NR_003278.3).

\section{Western blot analysis}

Cells were lysed in radioimmunoprecipitation assay (RIPA) buffer (50 mM Tris- $\mathrm{HCl}$ [pH 7.4], $150 \mathrm{mM} \mathrm{NaCl}, 1 \%$ Triton 
X-100, $1 \mathrm{mM}$ ethylene glycol tetra acetic acid, $1 \mathrm{mM}$ ethylenediaminetetraacetic acid, $0.1 \%$ sodium dodecyl sulfate [SDS], $1 \%$ sodium deoxycholate, $1 \mathrm{mM} \mathrm{Na} \mathrm{VO}_{4}, 1 \mathrm{mM}$ sodium fluoride, $1 \mathrm{mM}$ phenyl methyl sulfonyl fluoride, and protease inhibitor cocktail). After 30-minute incubation on ice, the lysates were centrifuged at $14,000 \mathrm{rpm}$ for 20 minutes at $4^{\circ} \mathrm{C}$. The protein concentration was measured with a bicinchoninic acid (BCA) protein assay kit (Pierce Chemical Co., Rockford, IL, USA). Protein samples were separated using sodium dodecyl sulfate-polyacrylamide electrophoresis, transferred to polyvinylidene fluoride membranes, and immunoblotted using the following antibodies [19]: MyHC (MF20), myogenin (sc-12732, Santa Cruz Biotechnology, Dallas, TX, USA), mineralocorticoid receptor (MR) (ab64457, Abcam, Cambridge, MA, USA), and $\beta$-tubulin (T2200, Sigma-Aldrich).

\section{Viability assay}

Cell viability was measured using cell counting kit-8 (CCK-8; Dojindo, Kumamoto, Japan), in accordance with the manufacturer's instructions. Briefly, $10 \mu \mathrm{L}$ of WST-8 dye (2-(2-methoxy-4-nitrophenyl)-3-(4-nitrophenyl)-5-(2,4-disulfophenyl)$2 \mathrm{H}$-tetrazolium, monosodium salt) was added to each well of a 96-well plate [19]. The suspension was incubated for 1 hour and the absorbance was then read at $450 \mathrm{~nm}$ with a reference wavelength of $650 \mathrm{~nm}$ using a microplate reader (SPECTRAmax 340PC, Molecular Devices, Palo Alto, CA, USA).

\section{Migration assay}

The chemotaxis assay was performed in a Boyden chamber system using a transwell with an 8 - $\mu$ m-pore size polycarbonate membrane (Corning, NY, USA). Cells were seeded onto the inner chamber at a density of $8 \times 10^{4}$ cells per transwell in DMEM with $0.2 \% \mathrm{FBS}$ and then exposed to aldosterone in the outer chamber for 5 hours [18]. The cells on the inner membrane were then completely removed by wiping with a cotton swab, while the cells on the lower surface of the membrane were fixed in $4 \%$ PFA and stained with crystal violet. To count the number of $\mathrm{C} 2 \mathrm{C} 12$ cells, the stained $\mathrm{C} 2 \mathrm{C} 12$ cells were photographed under cellSens Standard BX53 software (Olympus, Tokyo, Japan), and counted using Image $\mathbf{J}$ software (National Institutes of Health, Bethesda, MD, USA).

\section{Measurement of intracellular reactive oxygen species levels}

The intracellular reactive oxygen species (ROS) content was measured using the chloromethyl derivative of $2^{\prime}, 7^{\prime}$-dichloroflu- orescein diacetate (CM-H ${ }_{2}$ DCFDA, C6827, Invitrogen). Cells were washed with serum-free DMEM and incubated with 10 $\mu \mathrm{M} \mathrm{CM}-\mathrm{H}_{2} \mathrm{DCFDA}$ at $37^{\circ} \mathrm{C}$ for 30 minutes in the dark at $5 \%$ $\mathrm{CO}_{2}$. Then, cells were washed with PBS and visualized using a fluorescent microscope (Carl Zeiss, Jena, Germany). Fluorescence intensity was also read at an excitation wavelength of 490 $\mathrm{nm}$ and emission wavelength of $520 \mathrm{~nm}$ using a microplate reader (Infinite 200PRO, Tecan Life Sciences, Zürich, Switzerland).

\section{Ligand and receptor binding assay}

Recombinant aldosterone was coated onto the wells of Maxisorp 96-well microtiter plates and incubated for 18 hours at $4^{\circ} \mathrm{C}$. Each well was washed three times with PBST (0.1\% Tween-20 in PBS). Thereafter, the plates were blocked with $1 \%$ BSA in PBST for 2 hours. Cell lysates were added to the plates and incubated for 2 hours. The wells were then washed three times [20]. Preparations of the MR antibody (sc-53000, Santa Cruz Biotechnology) in blocking solution were added to the plates and allowed to react at $4^{\circ} \mathrm{C}$ overnight. After washing, horseradish peroxidase-linked antibody (Cell Signaling Technology) was added and the lysates were incubated for 2 hours; this was followed by five rounds of washes. The reaction was developed with $100 \mu \mathrm{L}$ of TMB substrate solution and stopped with 100 $\mu \mathrm{L}$ of $1 \mathrm{~N} \mathrm{H}_{2} \mathrm{SO}_{4}$. The absorbance in the microtiter plates was measured at $450 \mathrm{~nm}$ using a microplate reader (Infinite 200 PRO, Tecan Life Sciences).

\section{Statistical analysis}

All data are expressed as mean \pm standard error of the mean from at least three independent experiments with triplicate measurements, unless otherwise specified. The significance of differences among three or more groups was tested by analysis of variance (ANOVA) with post hoc analysis with Tukey's honest significance test, and differences between two groups were assessed using the Mann-Whitney $U$ test [19]. All statistical analyses were performed using SPSS version 18.0 (SPSS Inc., Chicago, IL, USA). $P<0.05$ was considered statistically significant.

\section{RESULTS}

\section{In vitro effects of recombinant aldosterone on muscle differentiation}

The $\mathrm{C} 2 \mathrm{C} 12$ myoblasts were differentiated into mature myotubes in the presence or absence of recombinant aldosterone. Aldosterone markedly suppressed in vitro myogenesis in a dose-depen- 

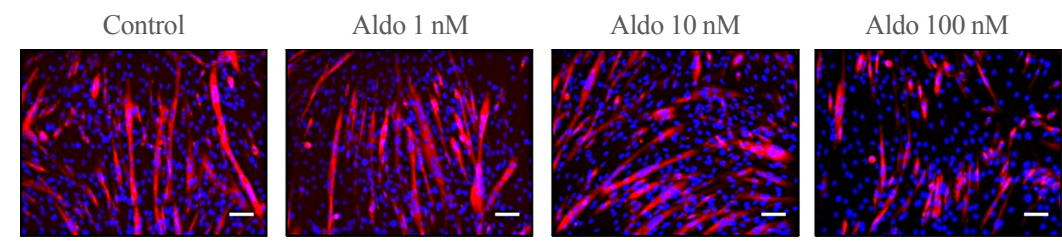

Aldo 1,000 nM
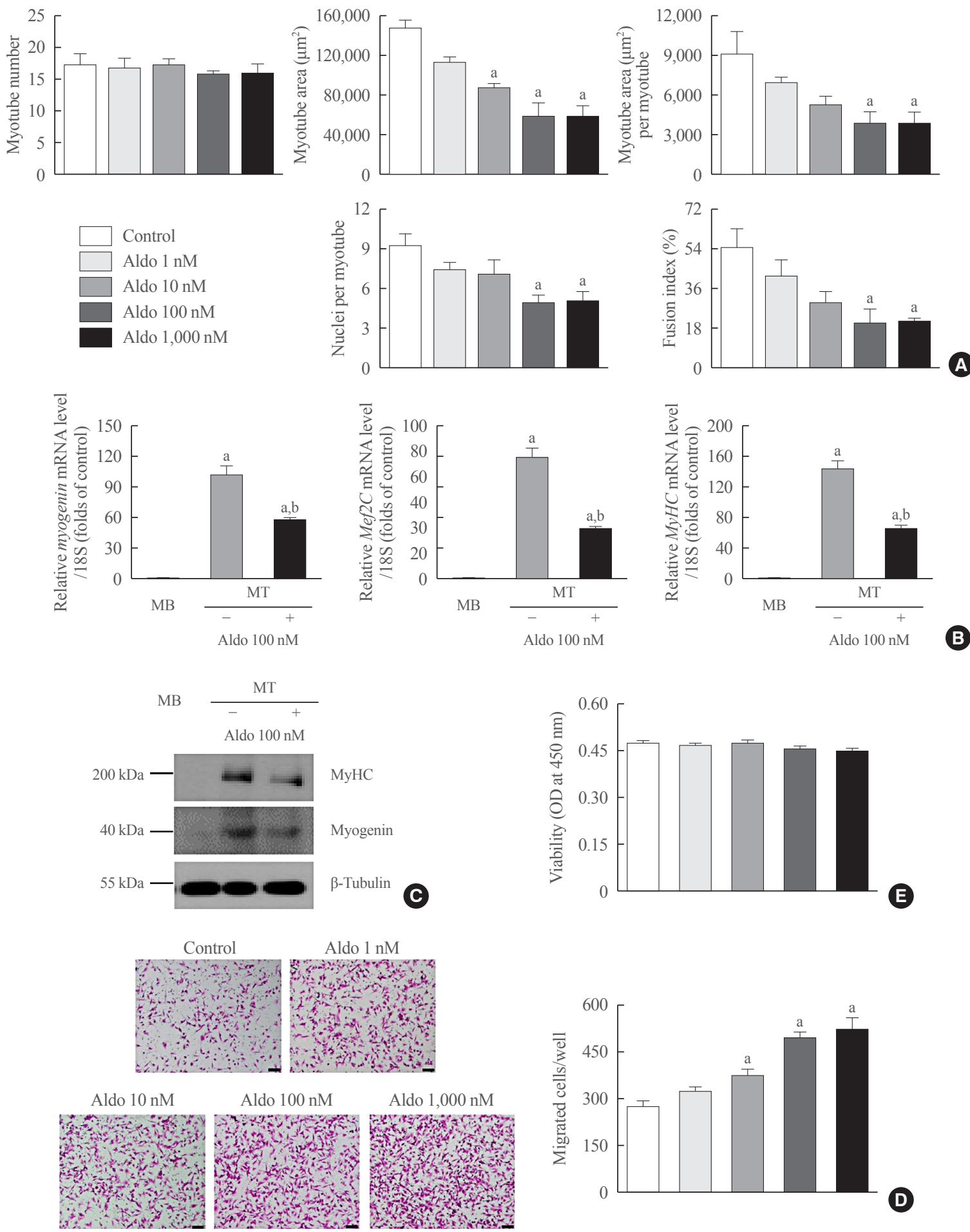

Fig. 1. Recombinant aldosterone (Aldo) inhibits in vitro myogenesis.

(Continued to the next page) 
Fig. 1. Continued. (A) Mouse $\mathrm{C} 2 \mathrm{C} 12$ myoblasts (MBs) were differentiated into myotubes (MTs) with $2 \%$ horse serum after exposure to the indicated concentrations of recombinant Aldo for 3 days. MTs were stained with anti-myosin heavy chain (MyHC) antibody while nuclei were counterstained with 4,6-diamidino-2-phenyindole (DAPI). Quantitative results per field are presented $(n=4)$. (B) Quantitative reversetranscription polymerase chain reaction and (C) Western blot analyses of myogenin, myocyte enhancer factor 2C (Mef2C), and/or MyHC in $\mathrm{C} 2 \mathrm{C} 12$ cells without or with $2 \%$ horse serum in the presence of $100 \mathrm{nM}$ Aldo for 3 days $(n=3)$. (D) The directional migration and (E) viability of mouse $\mathrm{C} 2 \mathrm{C} 12 \mathrm{MBs}$ were assessed by a Boyden chamber system and cell counting kit-8 (CCK-8) assay after exposure to the indicated concentrations of Aldo for 6 and 24 hours, respectively $(n=5)$. Scale bars: $100 \mu \mathrm{m}$ (A) and $50 \mu \mathrm{m}$ (D). Data are expressed as mean \pm standard error of the mean. OD, optical density. ${ }^{a} P<0.05$ vs. control or $\mathrm{MB} ;{ }^{\mathrm{b}} P<0.05$ vs. untreated MT.

dent manner, as confirmed by the decrease of myotube area, myotube area per myotube, nucleus number per myotube, and fusion index (Fig. 1A). Consistent with these findings, mRNA and protein expression levels of myogenic differentiation markers, such as myogenin, Mef2C, and/or MyHC, were significantly reduced by recombinant aldosterone (Fig. 1B, C, respectively). When we investigated the possible effects of aldosterone on other muscle biology, it was shown to stimulate the migration of C2C12 myoblasts (Fig. 1D) without affecting the viability (Fig. 1E). These results indicate that aldosterone plays a detrimental role in muscle metabolism mainly by inhibiting muscle differentiation.

\section{The inhibitory effects of aldosterone on myogenesis are mediated by increased intracellular ROS}

To determine the degree of intracellular ROS generation by recombinant aldosterone, we measured $\mathrm{CM}-\mathrm{H}_{2} \mathrm{DCFDA}$ content in the presence or absence of aldosterone during muscle differentiation. Aldosterone dose-dependently increased intracellular ROS levels in myotubes, and the maximum effect of a 1.9-fold increase was observed at $100 \mathrm{nM}$ aldosterone (Fig. 2A). Interestingly, pretreatment with NAC, a potent biological thiol antioxidant, significantly reduced aldosterone-induced stimulation of intracellular ROS generation in myotubes (Fig. 2B). Furthermore, NAC markedly reversed the decrease of myotube area, myotube area per myotube, nucleus number per myotube, and fusion index due to aldosterone (Fig. 2C), supporting the importance of oxidative stress in the suppression of myogenesis by recombinant aldosterone treatment.

\section{MR is the major receptor for aldosterone in muscle cells}

Because MR is known as a receptor for aldosterone in various types of cells $[21,22]$, we focused on the MR mediating the action of aldosterone in muscle cells. A binding affinity experiment using enzyme-linked immunosorbent assay (ELISA) indicated that the amount of aldosterone-associated MR increased as the amount of $\mathrm{C} 2 \mathrm{C} 12$ myoblast lysate increased (Fig. 3A). Western blot analyses also showed the increased expression of
MR during myogenesis (Fig. 3B). Importantly, pretreatment of C2C12 myoblasts with an MR inhibitor, eplerenone, in differentiation media blocked aldosterone-stimulated intracellular ROS generation (Fig. 3C) and markedly attenuated the suppression of in vitro myogenesis by aldosterone treatment (Fig. 3D). These findings indicate that aldosterone exerts its functions in muscle cells via MR and oxidative stress.

\section{DISCUSSION}

We have shown here that aldosterone inhibits in vitro myogenesis by stimulating intracellular ROS generation, which was reversed by NAC treatment. Furthermore, aldosterone and MR were shown to interact in muscle cells, and eplerenone blocked aldosterone-induced oxidative stress and myogenesis. These findings support the hypothesis that hypersecretion of aldosterone, like PA, directly contributes to muscular deterioration and suggest that antioxidants and/or MR antagonists could be an effective therapeutic option to reduce the risk of sarcopenia in these patients.

To investigate the possible mechanisms underlying the detrimental role of aldosterone in muscle metabolism, we focused on changes in redox signaling. In healthy skeletal muscle, the production of oxidant species is matched with the removal by exogenous or endogenous antioxidant molecules [23,24]. However, an imbalance of these processes in certain pathological conditions results in the loss of normal redox equilibrium, which is referred to as "oxidative stress" [25]. Various findings have indicated that the oxidative stress can lead to mitochondrial dysfunction, increase of myonuclear apoptosis and ubiquitin proteasome activity, autophagy deregulation, altered mineral homeostasis, and excitation-contraction uncoupling, all of which are involved in the decrease in muscle mass and quality $[23,26,27]$. Notably, dysregulation of the renin-angiotensin-aldosterone system (RAAS) damages several organs via oxidative stress mainly from nicotinamide adenine dinucleotide phosphate oxidase-derived ROS overproduction [28-30]. Based on this background, we investigated whether aldosterone increased 

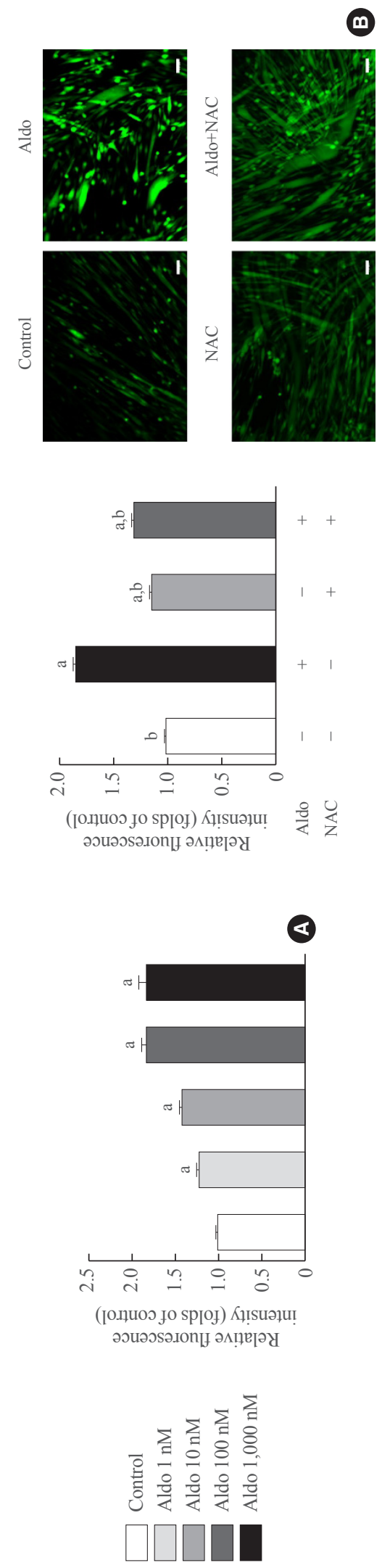
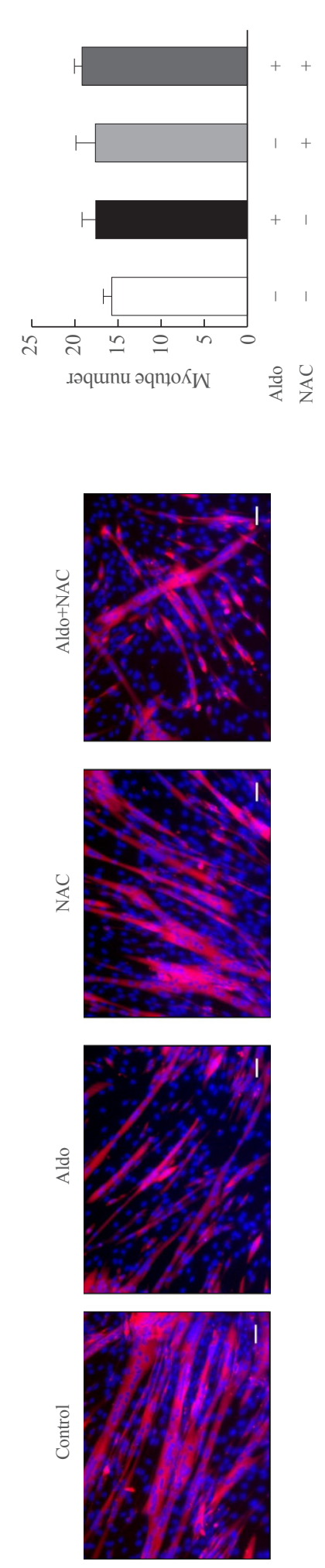

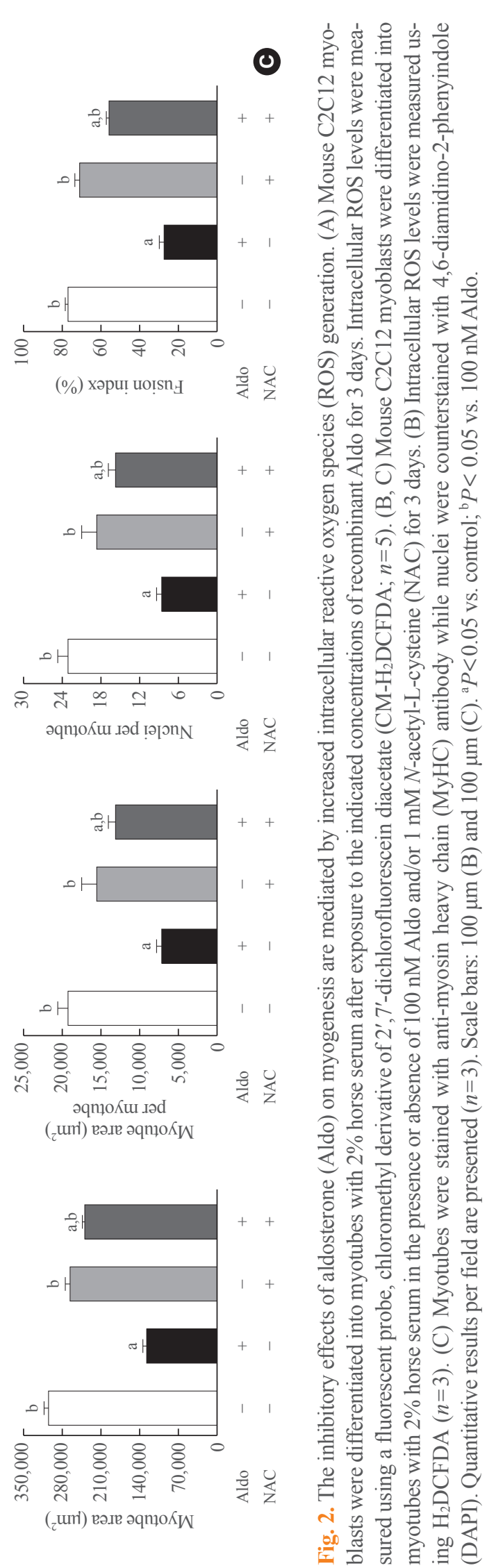

Copyright (C) 2021 Korean Endocrine Society 

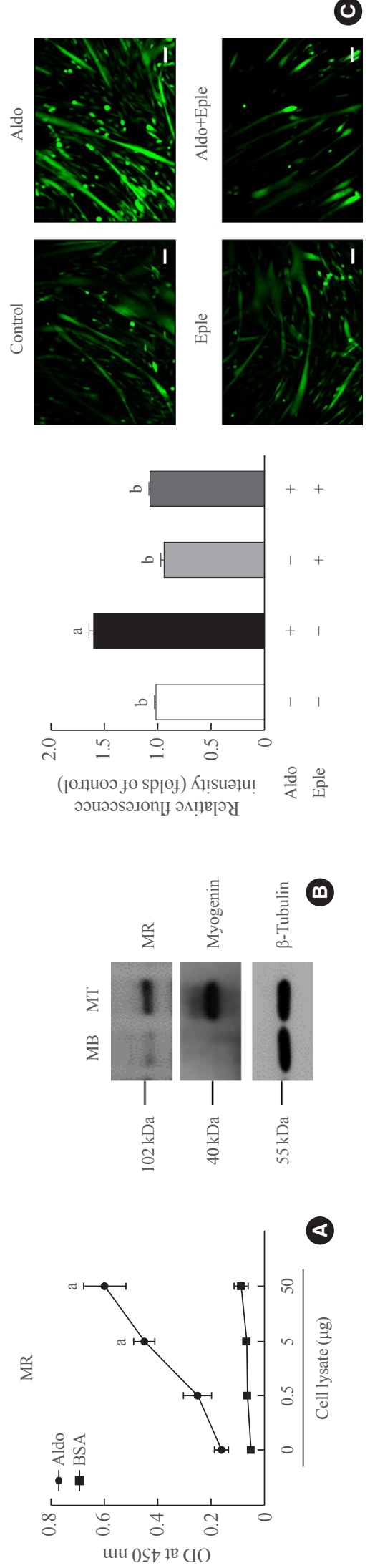
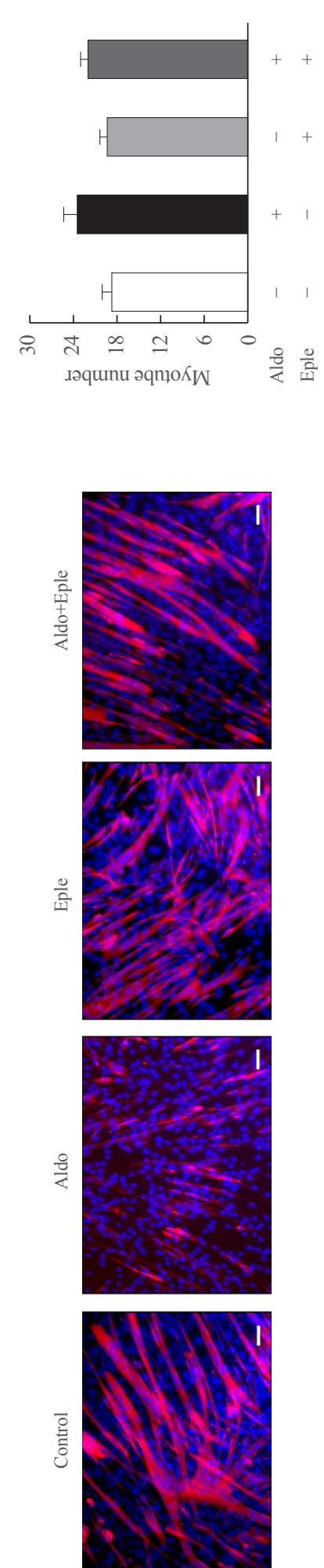

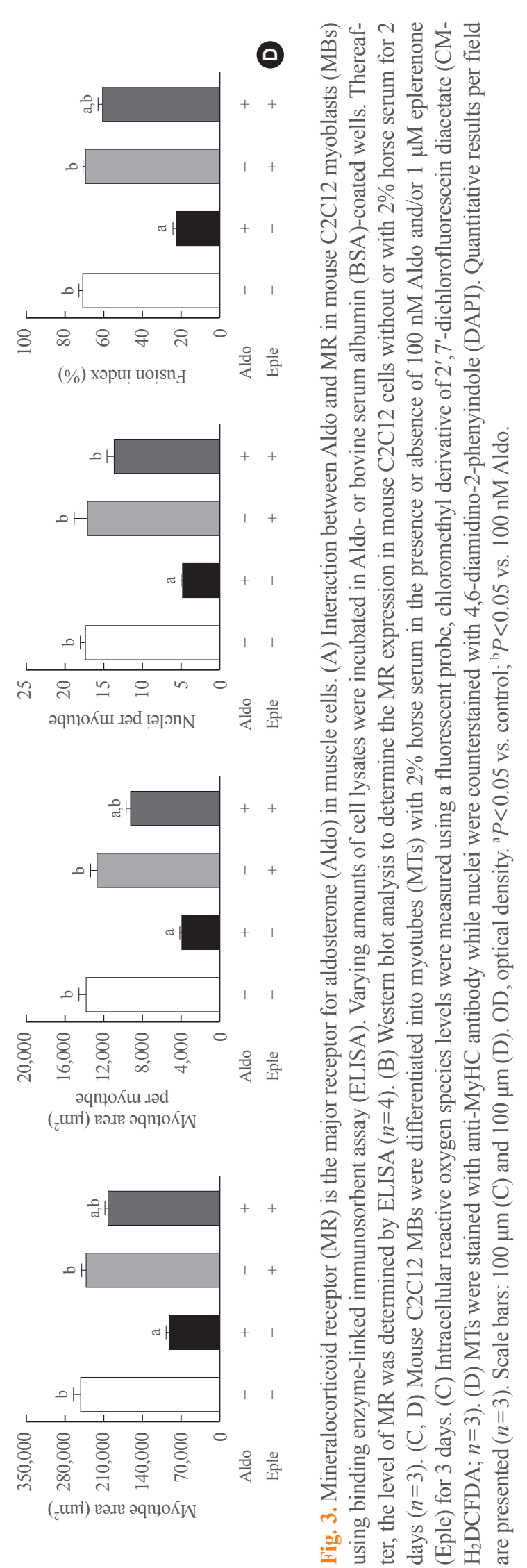

www.e-enm.org

871 
intracellular ROS production in muscle cells and antioxidants attenuated the impairment of myogenesis due to aldosterone. Our results demonstrated the importance of oxidative stress as a key modulator of the effect of aldosterone on muscle metabolism.

Aldosterone is a final hormone in the RAAS and exerts its effects by binding to MR [20]. Although the interaction between aldosterone and MR is mainly implicated in sodium and potassium homeostasis in the kidneys and the resultant blood pressure control, various tissues have been shown to be under the influence of a local RAAS [31,32]. We also reported that MR was strongly expressed in myotubes, consistent with a previous report [33], and eplerenone completely eliminated the effects of aldosterone on intracellular ROS generation and myogenesis. These results indicate that the interruption of RAAS via MR antagonists would be beneficial to maintain muscle health, in addition to the known role in reducing cardiovascular risks $[10,11]$.

Several points require discussion for the appropriate interpretation of our data. Firstly, although intracellular ROS levels in myotubes were dose-dependently increased by aldosterone treatment from $1 \mathrm{nM}(36 \mathrm{ng} / \mathrm{dL})$, which is the typical aldosterone concentration difference in subjects with and without PA, maximal detrimental effects on muscle biology were observed at $100 \mathrm{nM} \mathrm{(3,600} \mathrm{ng/dL)} \mathrm{aldosterone;} \mathrm{therefore,} \mathrm{using} 100 \mathrm{nM}$ aldosterone was appropriate for our study. Similarly, we acknowledge inevitable differences between in vitro environments and human systems. Secondly, spironolactone binds not only to MR but also to progestogen and androgen receptors [34]; therefore, to adequately investigate links between aldosterone and MR in muscle, we used eplerenone, a more selective MR antagonist than spironolactone. Thirdly, our results showing increased migration of $\mathrm{C} 2 \mathrm{C} 12$ myoblasts, despite suppressed muscle differentiation, by aldosterone were interesting. While we speculate that this could be an effect to compensate for damaged muscle cells by aldosterone, specific mechanisms and implications must be elucidated in future work. Lastly, angiotensinconverting enzyme (ACE) inhibitors and angiotensin II receptor blockers (ARBs) are widely used in clinical practice to abolish the effects of RAAS [10]. Considering these medications may partially reduce blood aldosterone levels, the protective role of ACE inhibitors and ARBs in human muscle health is a highly interesting research topic.

As the clinical importance of sarcopenia grows with the progressing aging of society, many studies are being conducted to find and reverse the related risk factors. PA is attracting attention not only as the most common cause of secondary hyperten- sion, but also for the adverse outcomes in musculoskeletal systems $[13,35,36]$. In the present study, we demonstrated that aldosterone leads to reduced myogenesis by directly binding to its receptor and the resultant increase of intracellular ROS production in muscle cells. These results provide the possible underlying mechanism explaining the deleterious effects of excess aldosterone on muscle metabolism. More interventional clinical studies demonstrating the improvement of muscle health by adrenalectomy, antioxidants, or MR antagonist in PA are required to translate the experimental results to humans.

\section{CONFLICTS OF INTEREST}

No potential conflict of interest relevant to this article was reported.

\section{ACKNOWLEDGMENTS}

This study was supported by National Research Foundation of Korea (NRF) grants funded by the South Korean government (MSIT) (grant numbers: 2019R1A2C2006527 and 2021R1C1C2006842).

\section{AUTHOR CONTRIBUTIONS}

Conception or design: J.Y.L., S.J.P., B.J.K. Acquisition, analysis, or interpretation of data: J.Y.L., D.A.K., E.C., Y.S.L., S.J.P., B.J.K. Drafting the work or revising: J.Y.L., S.J.P., B.J.K. Final approval of the manuscript: J.Y.L., D.A.K., E.C., Y.S.L., S.J.P., B.J.K.

\section{ORCID}

Jin Young Lee https://orcid.org/0000-0001-9116-4614

So Jeong Park https://orcid.org/0000-0003-0839-3016

Beom-Jun Kim https://orcid.org/0000-0001-8591-1759

\section{REFERENCES}

1. Cruz-Jentoft AJ, Sayer AA. Sarcopenia. Lancet 2019;393: 2636-46.

2. Dao T, Green AE, Kim YA, Bae SJ, Ha KT, Gariani K, et al. Sarcopenia and muscle aging: a brief overview. Endocrinol Metab (Seoul) 2020;35:716-32.

3. Cruz-Jentoft AJ, Bahat G, Bauer J, Boirie Y, Bruyere O, Cederholm T, et al. Sarcopenia: revised European consensus 
on definition and diagnosis. Age Ageing 2019;48:16-31.

4. Kim YK, Yi SR, Lee YH, Kwon J, Jang SI, Park SH. Effect of sarcopenia on postoperative mortality in osteoporotic hip fracture patients. J Bone Metab 2018;25:227-33.

5. Anker SD, Morley JE, von Haehling S. Welcome to the ICD-10 code for sarcopenia. J Cachexia Sarcopenia Muscle 2016;7:512-4.

6. Rosenberg IH. Sarcopenia: origins and clinical relevance. J Nutr 1997;127(5 Suppl):990S-991S.

7. Kim SH, Shin MJ, Shin YB, Kim KU. Sarcopenia associated with chronic obstructive pulmonary disease. J Bone Metab 2019;26:65-74.

8. Tevosian SG, Fox SC, Ghayee HK. Molecular mechanisms of primary aldosteronism. Endocrinol Metab (Seoul) 2019; 34:355-66.

9. Funder JW, Carey RM, Mantero F, Murad MH, Reincke M, Shibata $\mathrm{H}$, et al. The management of primary aldosteronism: case detection, diagnosis, and treatment: an Endocrine Society Clinical Practice Guideline. J Clin Endocrinol Metab 2016;101:1889-916.

10. Chen ZW, Hung CS, Wu VC, Lin YH; TAIPAI study group. Primary aldosteronism and cerebrovascular diseases. Endocrinol Metab (Seoul) 2018;33:429-34.

11. Milliez P, Girerd X, Plouin PF, Blacher J, Safar ME, Mourad JJ. Evidence for an increased rate of cardiovascular events in patients with primary aldosteronism. J Am Coll Cardiol 2005;45:1243-8.

12. Burniston JG, Saini A, Tan LB, Goldspink DF. Aldosterone induces myocyte apoptosis in the heart and skeletal muscles of rats in vivo. J Mol Cell Cardiol 2005;39:395-9.

13. Kwak MK, Lee SE, Cho YY, Suh S, Kim BJ, Song KH, et al. The differential effect of excess aldosterone on skeletal muscle mass by sex. Front Endocrinol (Lausanne) 2019;10: 195.

14. Kwak MK, Lee JY, Kim BJ, Lee SH, Koh JM. Effects of primary aldosteronism and different therapeutic modalities on glucose metabolism. J Clin Med 2019;8:2194.

15. Aagaard NK, Andersen H, Vilstrup H, Clausen T, Jakobsen J, Dorup I. Muscle strength, Na, K-pumps, magnesium and potassium in patients with alcoholic liver cirrhosis: relation to spironolactone. J Intern Med 2002;252:56-63.

16. Lastra G, Whaley-Connell A, Manrique C, Habibi J, Gutweiler AA, Appesh L, et al. Low-dose spironolactone reduces reactive oxygen species generation and improves insulinstimulated glucose transport in skeletal muscle in the TG(mRen2)27 rat. Am J Physiol Endocrinol Metab 2008;
295:E110-6.

17. Kim DA, Park SJ, Lee JY, Kim JH, Lee S, Lee E, et al. Effect of CCL11 on in vitro myogenesis and its clinical relevance for sarcopenia in older adults. Endocrinol Metab (Seoul) 2021;36:455-65.

18. Park SJ, Lee JY, Lee SH, Koh JM, Kim BJ. SLIT2 inhibits osteoclastogenesis and bone resorption by suppression of Cdc42 activity. Biochem Biophys Res Commun 2019;514: 868-74.

19. Lee JY, Park SJ, Kim DA, Lee SH, Koh JM, Kim BJ. Muscle-derived lumican stimulates bone formation via integrin $\alpha 2 \beta 1$ and the downstream ERK signal. Front Cell Dev Biol 2020;8:565826.

20. Kim BJ, Lee YS, Lee SY, Baek WY, Choi YJ, Moon SA, et al. Osteoclast-secreted SLIT3 coordinates bone resorption and formation. J Clin Invest 2018;128:1429-41.

21. Gorini S, Kim SK, Infante M, Mammi C, La Vignera S, Fabbri A, et al. Role of aldosterone and mineralocorticoid receptor in cardiovascular aging. Front Endocrinol (Lausanne) 2019;10:584.

22. Inthachart K, Manotham K, Eiam-Ong S, Eiam-Ong S. Aldosterone rapidly enhances levels of the striatin and caveolin-1 proteins in rat kidney: the role of the mineralocorticoid receptor. Endocrinol Metab (Seoul) 2019;34:291-301.

23. Szentesi P, Csernoch L, Dux L, Keller-Pinter A. Changes in redox signaling in the skeletal muscle with aging. Oxid Med Cell Longev 2019;2019:4617801.

24. Powers SK, Morton AB, Ahn B, Smuder AJ. Redox control of skeletal muscle atrophy. Free Radic Biol Med 2016;98: 208-17.

25. Droge W. Free radicals in the physiological control of cell function. Physiol Rev 2002;82:47-95.

26. Abrigo J, Elorza AA, Riedel CA, Vilos C, Simon F, Cabrera $\mathrm{D}$, et al. Role of oxidative stress as key regulator of muscle wasting during cachexia. Oxid Med Cell Longev 2018; 2018:2063179.

27. Mecocci P, Fano G, Fulle S, MacGarvey U, Shinobu L, Polidori MC, et al. Age-dependent increases in oxidative damage to DNA, lipids, and proteins in human skeletal muscle. Free Radic Biol Med 1999;26:303-8.

28. Cooper SA, Whaley-Connell A, Habibi J, Wei Y, Lastra G, Manrique $\mathrm{C}$, et al. Renin-angiotensin-aldosterone system and oxidative stress in cardiovascular insulin resistance. Am J Physiol Heart Circ Physiol 2007;293:H2009-23.

29. Fanelli C, Zatz R. Linking oxidative stress, the renin-angiotensin system, and hypertension. Hypertension 2011;57: 
373-4

30. Brown NJ. Aldosterone and vascular inflammation. Hypertension 2008;51:161-7.

31. Hawkins UA, Gomez-Sanchez EP, Gomez-Sanchez CM, Gomez-Sanchez CE. The ubiquitous mineralocorticoid receptor: clinical implications. Curr Hypertens Rep 2012;14: 573-80.

32. Gomez-Sanchez EP. Brain mineralocorticoid receptors: orchestrators of hypertension and end-organ disease. Curr Opin Nephrol Hypertens 2004;13:191-6.

33. Chadwick JA, Hauck JS, Lowe J, Shaw JJ, Guttridge DC, Gomez-Sanchez CE, et al. Mineralocorticoid receptors are present in skeletal muscle and represent a potential therapeutic target. FASEB J 2015;29:4544-54.

34. Delyani JA, Rocha R, Cook CS, Tobert DS, Levin S, Roniker B, et al. Eplerenone: a selective aldosterone receptor antagonist (SARA). Cardiovasc Drug Rev 2001;19:185-200.

35. Kim BJ, Lee SH, Koh JM. Bone health in adrenal disorders. Endocrinol Metab (Seoul) 2018;33:1-8.

36. Kim BJ, Kwak MK, Ahn SH, Kim H, Lee SH, Koh JM. Lower trabecular bone score in patients with primary aldosteronism: human skeletal deterioration by aldosterone excess. J Clin Endocrinol Metab 2018;103:615-21. 